\title{
Evaluation of the Amount and Type of Microorganisms in Tooth Brushes and Miswak after Immediate Brushing
}

\author{
Rita Khounganian BDS, MSc, PhD $^{1}$, Abdul-Aziz Alwakeel BDS, SBOMP ${ }^{2^{*}}$, Abdulhakim \\ Albadah BSc, $\mathrm{MSc}^{3}$, Nassr Almaflehi BSc, MSc ${ }^{4}$ \\ ${ }^{1}$ Professor, Department of Oral Medicine and Diagnostic Sciences, College of Dentistry, King Saud University, \\ Riyadh, Saudi Arabia \\ ${ }^{2}$ Saudi Board Resident in Oral Medicine and Pathology, Division of Oral Medicine and Diagnostic Sciences, \\ College of Dentistry, King Saud University, Riyadh, Saudi Arabia \\ ${ }^{3}$ Microbiologist, College of Dentistry, King Saud University, Riyadh, Saudi Arabia \\ ${ }^{4}$ Biostatistician, College of Dentistry, King Saud University, Riyadh, Saudi Arabia
}

*Corresponding Author: Abdul-Aziz Alwakeel, Saudi Board Resident in Oral Medicine and Pathology, Division of Oral Medicine and Diagnostic Sciences, College of Dentistry, King Saud University, Riyadh, Saudi Arabia Email: alwakeelaziz@gmail.com

\section{Abstract}

Objectives: To evaluate the amount and type of microorganisms attached on tooth brushes with and without fluoride tooth paste and miswak after immediate brushing.

Methods: A Cross sectional study was conducted on 29 randomly selected participants. They were instructed to brush their teeth once/week; with tooth brush alone, tooth brush with fluoride tooth paste and miswak alone. The samples were immediately processed for microbiological and scanning electron microscopic investigations. The frequency distribution, mean and standard deviation, were calculated the level of statistical significant difference was set at $p \leq 0.05$.

Results: All the participants cleaned their teeth with tooth brushes alone on the first day while 27 only used tooth brushes with fluoride tooth paste on the second week, whereas 24 participants used the miswak alone. The predominant type of bacteria revealed in the present study was Gram -ve/+ve cocci and Gram-ve/+ve bacilli. There was a direct correlation between tooth brushing methods used and type of bacteria. Friedman repeated measurement test revealed significant differences between the three brushing methods with $p=.000$. The highest count of bacterial number was found in the miswak group and the lowest count in the tooth brush with fluoride tooth paste.

Conclusion: The tooth brushes with or without fluoride tooth paste were carrying bacteria after each use including miswak. The tooth brushes should be thoroughly washed with sterile water and disinfected after each use. Additionally, the miswak used tip for brushing should be properly cut and trimmed after each single use.

Keywords: Tooth brushing, Fluoride tooth paste, Miswak, Bacteria, Microorganisms

\section{INTRODUCTION}

The oral cavity is the major portal of entry, source, and site of many diseases affecting the general health status. Well-being and quality of life, which is measured along functional, psychosocial, and economic dimensions, is related to oral health (1). Good oral hygiene is considered as a sign for good oral and systemic health. According to Almas \& Almas "acceptable level of plaque control, from where periodontal disease cannot be initiated or progressed is the ultimate desire of oral and dental professionals"(2).

At birth, no microorganisms are shown in the oral cavity because the fetus is developed in clear conditions, there is large number of microorganisms developing in the oral cavity in the first days of life such as ; Streptococcus, 
Staphylococcus, Neisseria, Candida, Lactobacillus, Veillonella and coliforms. The streptococci mutans bacteria which is the primary responsible etiological factor for dental caries in humans, usually appears following primary tooth eruption, as it survives on hard surfaces (3).

The Tooth brush is considered one of the most common oral hygiene aids. It is used to promote oral hygiene health and prevent oral diseases. The contamination of tooth brushes occur by the microorganisms present in the oral cavity. The Retention and survival of micro-organisms on tooth brushes after brushing represents a possible cause of mouth recontamination. The probability of these tooth brushes being associated with transmission of severe health problems have been well documented, affecting both oral and general health $(4,5)$.

Miswak is a traditional chewing stick taken from the roots of special tree, twigs, and stem of Salvadora persica. It has been used as a natural method for brushing the teeth in many countries world wide for thousands of years (1). Using the miswak adjunct to tooth brushing have shown significant improvement in plaque score and gingival health, but the miswak cannot replace the toothbrush (6). Large reduction in the numbers of streptococcus mutans was found in groups using miswak, apparently the counts were more reduced in groups using both miswak and a conventional toothbrush (7).

\section{OBJECTIVES}

The present research is undertaken to evaluate the amount and type of microorganisms attached on tooth brushes with and without fluoride tooth paste and miswak after immediate brushing.

\section{Materials AND MethodS}

A Cross sectional study was conducted on 29 randomly selected participants. They were all provided with identical conventional tooth brushes, fluoride tooth paste and Miswak and were efficiently instructed to brush their teeth with one of the following three methods once/week on 3 consecutive Mondays; with tooth brush alone, tooth brush with fluoride tooth paste and miswak alone. The samples were immediately placed in laboratory vials containing sterile water and accordingly processed for microbiological and scanning electron microscopic investigations using the Jeol - 6360 LV SEM. The study was approved and supported by Research Center College of Dentistry, King Saud University (CDRC registration no. \# IR0179).

\section{Microbiological Methodology}

Brain heart infusion and blood agar were used as the media. They were prepared according to the manufacturer's instructions (Oxoid LTD, England). Blood agar and brain heart infusion agar plates were both aerobically incubated (Thermo electron / Germany) for 48h. After incubation, the bacterial growth on the brain heart infusion agar plates were counted using the colony counter (Reicher - Jung / USA) and the bacteria from the blood agar plates were in turn smeared on the slides for gram staining to identify their types.

\section{Statistical AnalysiS}

The collected data was entered and analysed using SPSS ${ }^{\circ}$ Statistics, Version 20 (IBM Corp., Armonk, NY, USA). Descriptive statistics were used to calculate the frequency distribution, mean, standard deviation and median. For the total number of Bacteria in the different groups, Friedman repeated measurement test was used. The level of statistical significant difference was set at $\mathrm{p} \leq 0.05$.

\section{RESUlts}

All 29 participants cleaned their teeth with the provided tooth brushes alone on the first day while only 27 of them used tooth brushes with fluoride tooth paste on the second week, whereas 24 participants used the miswak alone on the third week.

Bacterial growth on blood and brain heart infusion agar media was carried out to compare the type and number of bacteria respectively found on the tooth brush alone, tooth brush \& tooth paste with fluoride and miswak groups as shown in figure 1. Blood agar have shown similar types of bacteria on tooth brushes with tooth paste and miswak, while brain heart infusion agar revealed the highest number of bacterial count in the miswak group and the least was detected in the tooth brush with tooth paste (Fig. 1). 
Evaluation of the Amount and Type of Microorganisms in Tooth Brushes and Miswak after Immediate Brushing

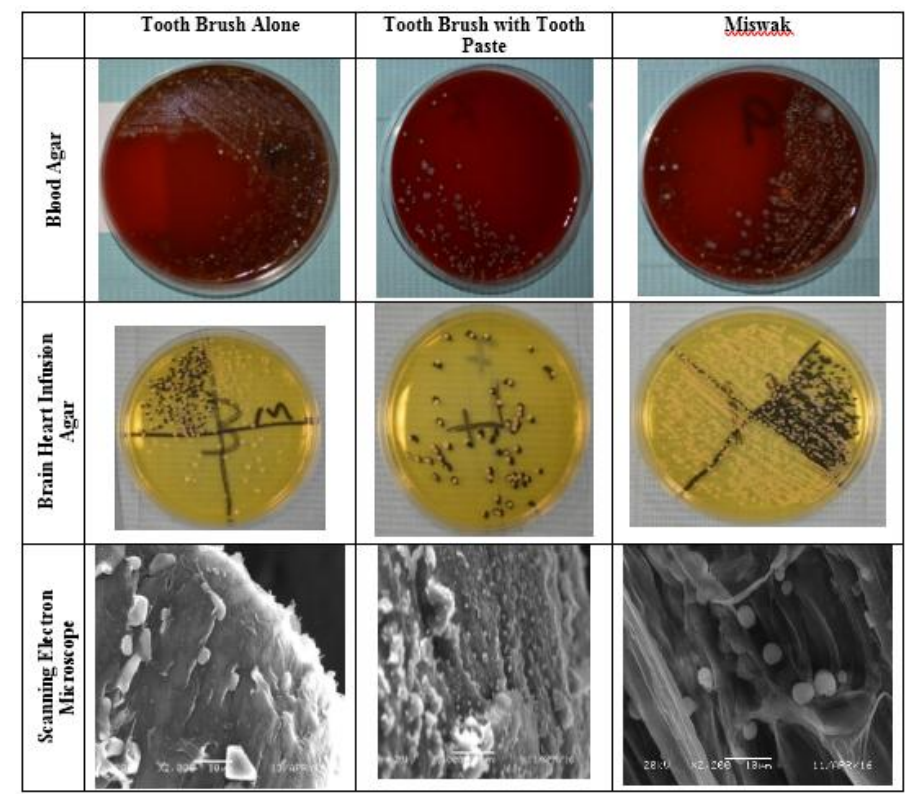

Figure1. Bacterial growth detected on tooth brush, tooth brush with tooth paste and Miswak with blood agar, brain heart infusion agar and SEM

Regarding the type of bacteria detected on the blood agar for each method used by the participants, various types of bacteria were mostly observed in tooth brush with tooth paste and miswak in equal amounts and the least on tooth brush alone. Both Gram +ve and Gram -ve cocci values were higher in the tooth brush alone groups while Gram +ve was less seen in the tooth brush with tooth paste group and the Gram -ve cocci were lowest in the miswak group. Gram +ve bacilli was high in tooth brush with tooth paste but low in miswak, whereas the Gram -ve bacilli was high in miswak but low in tooth brush with tooth paste (Fig.2).

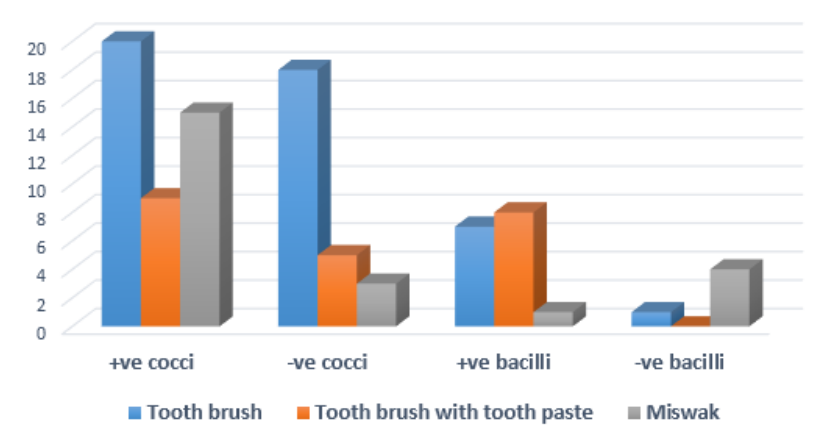

Figure2. Type of microorganisms detected in all participants using different brushing methods

The Scanning Electron Microscopic findings revealed bacteria in the form of cocci and bacilli in all groups (Fig.1). Bacteria were also observed attached to the bristles of a newly unused miswak (Fig. 3)

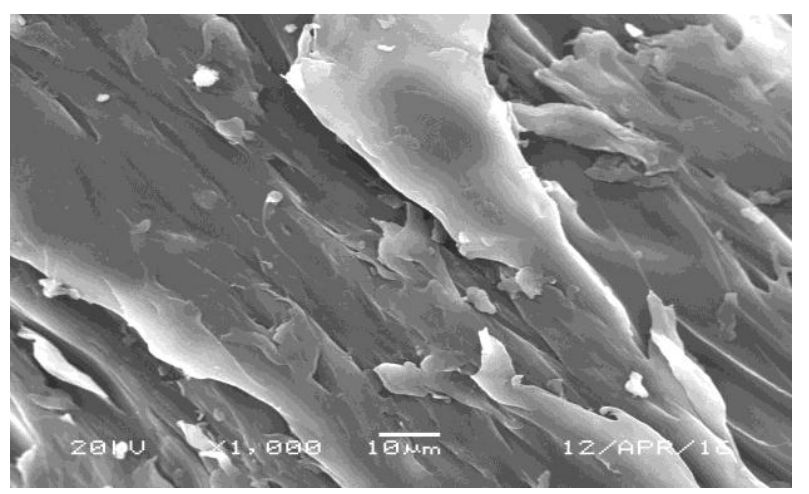

Figure3. Scanning electron microscopic findings of bacteria attached to the bristles of new miswak 
To calculate the total number of Bacteria in the different groups, Friedman repeated measurement test was used. Significant differences were identified between the three brushing methods with $p=.000$ as shown in figure 4 and table 1 .

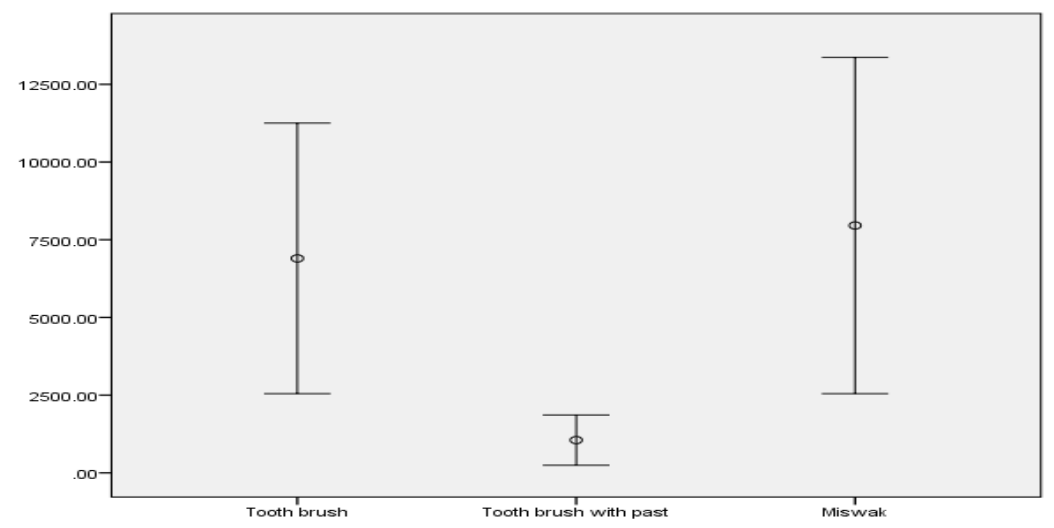

Figure4. Bacterial Count in the different brushing groups

There was a direct correlation between the tooth brushing methods used and amount of bacteria. The highest count of bacterial number was found in the miswak group and the lowest count was detected in the tooth brush with tooth paste as shown in table 1 .

Table1. Bacterial Count in the different brushing groups

\begin{tabular}{|l|c|c|c|}
\hline \multicolumn{1}{|c|}{ Bacterial Count } & Tooth Brush Alone & $\begin{array}{c}\text { Tooth Brush \& } \\
\text { Tooth Paste }\end{array}$ & Miswak \\
\hline Mean \pm SD & $7766.92 \pm 12379.7$ & $1179.46 \pm 2143^{*}$ & $7957.79 \pm 12809$ \\
\hline Median & 3813 & 5 & 3627 \\
\hline IQR & 4770 & 1819.75 & 3224 \\
\hline
\end{tabular}

* Statistical significance at $p \leq 0.05$

\section{DisCuSSION}

The methods available for the maintenance of oral health are mainly mechanical and chemical. Tooth brushing is the most common mechanical method used to control plaque (6). The increased awareness of the need for good dental health and the emphasis on preventive procedures have made the role of the tooth brush increasingly important (5). The documented literature revealed that tooth brushes can be a pool for the direct transmission of microorganisms along with a source for inoculation or re-establishment of microorganisms from infected to non-infected tissues $(3,6)$. Consequentially the present study was designed to evaluate the amount and type of microorganisms attached on tooth brushes with and without fluoride tooth paste and miswak after immediate brushing.

The present findings demonstrated bacterial growth on blood agar and brain heart infusion agar after a single use of the three different brushing methods. The scanning electron microscopy have supported the presence of bacteria on the toothbrush bristles supporting the earlier studies of Svanberg who observed a massive infection of mutans streptococci on toothbrushes within $24 \mathrm{~h}$ of use (8), whereas in contradistinction to Taji \& Rogers who reported no mutans streptococci from adult toothbrushes in a pilot study (9). The toothbrush with fluoride tooth paste have shown low counts of bacteria when compared with the tooth brush alone, this may explain the antimicrobial effect of the tooth pastes.

Time necessary for colonization is contradictory, varying from 1 to 30 days. According to Cesco et al. colonization of toothbrushes by mutans streptococci occurs in a short time period, since after a single tooth brushing, they found the development of the microorganism in $24 \%$ of the cases (10) supporting the present findings where colonization by Gram +ve \& Gram - ve bacteria was observed on bristles after one single use of tooth brush, tooth brush with tooth paste and even miswak use; similarly Svanberg reported the presence of mutans streptococci on toothbrushes (8).

Several authors have reported that bacterial contamination can be reduced by washing toothbrushes after use, and drying in aerated conditions (3). Caudry et al. have reported that a wet environment increases bacterial growth and 
cross contamination (11). Therefore, as time increases between one tooth brushing and another, more microorganism development can occur in the toothbrushes stored in a wet/moisture environment $(4,12)$.

Some investigators have recommended the need for toothbrush cleansing to reduce the number of microorganisms on the bristles, using various methods including boiling water, UV-radiation, microwave oven and chemical agents such as Listerine (13). Caudry et al. had suggested that immersion in Listerine, for $20 \mathrm{~min}$ is an efficient method of disinfection (11). Chandrdas et al, urged the need for disinfecting tooth brushes after each use, they reported that $3 \%$ garlic showed the highest efficacy followed by $0.05 \%$ Cetyl pyridinium chloride, $0.2 \%$ Chlorhexidine gluconate, $0.2 \%$ tea tree oil, and UV toothbrush sanitizing device (14). Moreover, Naik et al. stated that the use of chlorhexidine gluconate and sodium hypochlorite are efficient to decontaminate the tooth brushes (5); supporting the results of the present study where tooth brushes were found carrying bacteria after one single use and henceforth should be disinfected or discarded at regular intervals for public welfare.

American Dental Association (ADA) recommends routine change of toothbrushes every three months (15). Many patients, however, reported psychological, economical, and environmental barriers to frequently changing their toothbrushes (14).

Cleansing teeth with contaminated tooth brush will do more harm than good. The oral cavity can be a source of contamination to toothbrushes, environment, hands, aerosol pollution, and storage containers. Bacteria which attach and accumulate on toothbrushes, grow and flourish in warm and moist conditions (4). Reinfection of the oral cavity is possible owing to injuries of the gingiva that can occur during tooth brushing. Tooth brushes that play a pivotal role in fighting against tooth decay can itself lead to dental caries, gingivitis, stomatitis as well as many other systemic diseases including infective endocarditis, arthritis, bacteremia and septicemia if not properly stored and maintained (16).

The selection of miswak for the present study was based on a number of factors. Miswak is a chewing stick commonly used in the Middle East region and many developing countries as a traditional toothbrush for oral hygiene (17). It has an acceptable taste, inexpensive (15) and has been reported to have anti-plaque and many therapeutic pharmacological properties (18). It is reported to be effective for the removal of dental plaque from tooth surfaces and interproximal sites (6), contrary to the conventional tooth brushing where it is relatively ineffective for the removal of interproximal plaque.

Surprisingly, despite the widespread use of miswak since ancient times, relatively little scientific attention has been paid to its oral health beneficial effects (19) despite the fact that the World health Organization in 1987, recommended and encouraged developing nations to use Miswak as an effective tool for oral hygiene particularly in areas where their use is customary $(20,21)$.

The value of chewing sticks is believed to be in their mechanical cleansing action (21). The custom use of miswak has also been proven to hinder the chemical development of dental plaque with an antimicrobial outcome against many types of oral bacteria (6). On the other hand, Haque \& Alsareii have identified more plaque formation and gingival bleeding in individuals who used chewing sticks in contrast with toothbrush users (1). Moreover, Gazi et al. reported that the toothbrush was more efficient as an oral hygiene aid than the miswak (22), in contradistinction to that stated by Al Otaibi et al. (21).

As far as the antimicrobial actions of miswak is concerned, the risk of dental caries identified was 9.35 times more in subjects using toothbrush than those using chewing sticks (23). However, the consideration of dental caries was beyond the scope of the present study and henceforth cannot suggest any effective equivalency of tooth brush and miswak relevancy to dental caries.

This might shed some light on the present findings where the miswak group had increased counts of bacteria when compared to the tooth brush with tooth paste but less count of bacteria when compared with the tooth brush alone group. According to Almas and Al Zied, miswak has an antibacterial effect on cariogenic bacteria and periodontal pathogens with an inhibitory action on dental plaque formation (7). Patel et al. concluded that miswak cannot replace the toothbrush, but can be used an adjunct to toothbrush, utilizing the mechanical efficacy of toothbrush and chemical effects of miswak (6).

Reports on the oral health of miswak users are contradictory and published literatures suggest 
that its therapeutic role in oral health needs to be verified before implementing into general practice of dentistry (2). Furthermore, the difference in topographic design of handles and bristles of miswak stick might be another reason of poor plaque control in miswak users. (24). Miswak bristles are apparently not similar to toothbrush bristles that are used to mechanically remove plaque from the tooth surfaces. Unlike a conventional toothbrush, the bristles of the miswak lie in the same long axis as its handle. The angulations in the toothbrush enable it to adapt more easily to the distal tooth surfaces, particularly on the posterior teeth (1). However, the techniques employed for removing plaque mechanically from outer surface and interproximal sites are similar with the toothbrush and the chewing stick, e.g., vertical and horizontal brushing. These techniques primarily depend upon the people's attitudes, knowledge, and manual dexterity. These mechanical differences were evident in our results.

Previous studies have revealed that freshly prepared miswak sticks have no cytotoxic effect on periodontal tissue. However, the same plant used even after 24 hours does contain harmful cytotoxic components and has potential to damage periodontal cells $(24,25)$. Based on these findings, we recommended cutting the used portion of the miswak after it has been used for one day and preparing a fresh part for the next use.

\section{CONCLUSiON}

The tooth brushes with or without fluoride tooth paste were carrying bacteria after each use including miswak. The tooth brushes should be thoroughly washed with sterile water and disinfected after each use. Additionally, the miswak used tip for brushing should be properly cut and trimmed after each single use.

\section{REFERENCES}

[1] Haque MM, Alsareii S. A review of the therapeutic effects of using miswak (Salvadora Persica) on oral health. Saudi Med J. 2015; 36 (5): $\quad 530 \quad-\quad 543 . \quad$ (doi: 10.15537/smj.2015.5.10785)

[2] Almas AK, Almas K. Miswak (Salvadora Persica Chewing Stick) and Its Role in Oral Health; an Update. J Pak Dent Assoc. 2013; 22 : $255-264$.

[3] Filho PN, Macari S, Faria G, Assed S, Yoko II. Microbial contamination of toothbrushes and their Decontamination. American Academy of Pediatric Dentistry. 2000; 22(5): 381 - 384.

[4] Karibasappa GN, Nagesh L, Sujatha, BK. Assessment of microbial contamination of toothbrush head: An in vitro study. Indian J Dent Res. 2011;22: 2 - 5 .

[5] Naik R, Ahmed-Mujib BR, Telagi N, Anil BS, Spoorthi BR. Contaminated tooth brushespotential threat to oral and general health. J Family Med Prim Care. 2015; 4: 444 - 448.

[6] Patel PV, Shruthi S, Kumar S. Clinical effect of miswak as an adjunct to tooth brushing on gingivitis. J Indian Soc Periodontol. 2012; 16: $84-88$.

[7] Almas K, Al-Zied Z. The Immediate Antimicrobial Effect of a Toothbrush and Miswak on Cariogenic Bacteria: A Clinical Study. The Journal of Contemporary Dental Practice. 2004; 5 (1): 15.

[8] Svanberg M. Contamination of toothpaste and toothbrush by Streptococcus mutans. Scan J Dent Res. 1978; 86: 412 - 414.

[9] Taji SS, Rogers AH. The microbial contamination of tooth brushes. A pilot study. Aust Dent J. 1998;43: 128 - 130.

[10] Cesco RT, Bignelli P, Santos CP, Ito IY. Toothbrushes: Evaluation of contamination level by streptococci of mutans group. 5th World Congress on Preventive Dentistry. Transamérica Hotel, São Paulo, Brazil, 1995; April 27 - 30: pp 103.

[11] Caudry SD, Klitorinos A, Chan ECS. Contaminated toothbrushes and their disinfection. J Can Dent Assoc. 1995; 61: 511516.

[12] Verran J, Leahy-Gilmartin AA. Investigations into the microbial contamination of toothbrushes. Microbios. 1996; 85: 231 - 238.

[13] Konidala U, Nuvvula S, Mohapatra A, Nirmala S. Efficacy of various disinfectants on microbially contaminated toothbrushes due to brushing. Contemp Clin Dent. 2011; 2: $302-$ 307.

[14] Chandrdas D, Jayakumar HL, Chandra M, Katodia L, Sreedevi A. Evaluation of antimicrobial efficacy of garlic, tea tree oil, cetyl pyridinium chloride, chlorhexidine, and ultraviolet sanitizing device in the decontamination of toothbrush. Indian J Dent. 2014; 5: 183 - 189.

[15] World Health Organization. Oral health surveys. Basic methods. Geneva: WHO. 1997.

[16] Michelle RF, Cindy LM. Toothbrush Contamination: A Review of the Literature. Nursing Research and Practice. 2012; volume 2012: Article ID 420630, 6 pages. (doi:10.1155/ 2012/42 0630) 
[17] Malik AS, Shaukat MS, Qureshi AA, Abdur R. Comparative effectiveness of chewing stick and tooth brush: A randomized clinical trial. N Amer J Med Sciences. 2014; 6 (7): 333 - 337.

[18] Mohammed SA, Khan JA. Antioxidant capacity of chewing stick miswak Salvadora persica. BMC Complement Alternative Med. 2013; 13: 40.

[19] Consensus Statement on Oral Hygiene. Int Dent J. 2000; 52: 139.

[20] World Health Organization. Prevention of diseases. Geneva: WHO. 1987; 60 - 61.

[21] Al Otaibi M, Al Harthy M, Söder B, Gustafsson A, Månsson BA. Comparative effect of chewing sticks and tooth brushing on plaque removal and gingival health. Oral Health Prev Dent. 2003; 1: $301-307$.
[22] Gazi M, Saini T, Ashri N, Lambourne A. Meswak chewing stick versus conventional tooth- brush as an oral hygiene aid. Clin Prev Dent. 1990;12: $19-23$.

[23] Ezoddini AF. The efficacy of Miswak (Salvadora Persica) in preventing dental caries. Health. 2010; 2: $499-503$.

[24] Almas K, Al-Bagieh N, Akpata ES. In vitro antibacterial effect of freshly cut and 1-month-old Miswak extracts. Biomedical Lett. 1997; 56: 145-149.

[25] Al-Obaida MI, Al-Essa MA, Asiri AA, Al-Rahla AA. Effectiveness of a 20\% Miswak extract against a mixture of Candida albicans and Enterococcus faecalis. Saudi Med J. 2010; 31: $640-643$.

Citation: Khounganian R, Alwakeel A, Albadah A, Almaflehi N. Evaluation of the Amount and Type of Microorganisms in Tooth Brushes and Miswak after Immediate Brushing. ARC Journal of Dental science. 2018; 3(1): 15-21. doi:dx.doi.org/ 10.20431/2456-0030. 0301005.

Copyright: (C) 2018 Authors. This is an open-access article distributed under the terms of the Creative Commons Attribution License, which permits unrestricted use, distribution, and reproduction in any medium, provided the original author and source are credited. 